Proceedings

\title{
Systematic Electro-Optical Study of Photodiodes in Intrinsic Material (Lowly Doped) with Backend Stack Optimization ${ }^{\dagger}$
}

\author{
Frederic Roger *, Ingrid Jonak-Auer, Olesia Synooka, Filip Segmanovic, Joni Mellin and \\ Helmut Hofstaetter
}

AMS AG, Device R\&D, Tobelbader Strasse 30, 8141 Premstaetten, Austria;

Ingrid.Jonak-Auer@ams.com (I.J.-A.); Olesia.Synooka@ams.com (O.S.); Filip.Segmanovic@ams.com (F.S.); Joni.Mellin@ams.com (J.M.); Helmut.Hofstaetter@ams.com (H.H.)

* Correspondence: Frederic.Roger@ams.com; Tel.: +43-3136-500-32095

+ Presented at the Eurosensors 2018 Conference, Graz, Austria, 9-12 September 2018.

Published: 26 November 2018

\begin{abstract}
This paper constitutes the analysis of the impact of low doped intrinsic p-type EPI thickness $(20 \mu \mathrm{m}$ and $30 \mu \mathrm{m})$ and bottom anti-reflective coating on the electrical and optical performance of various PIN photodiodes designs. The intrinsic p-type layer with a target resistivity of $400 \Omega \mathrm{cm}$ is an epitaxial layer (iEPI) grown on a low resistive substrate of $20 \mathrm{~m} \Omega \mathrm{cm}$. Optimization of the photodiode's spectral responsivity (for a specific wavelength) includes a Bottom AntiReflective Coating (BARC) layer deposited over the silicon surface. BARC thickness is optimized for $\lambda=425 \mathrm{~nm}, \lambda=750 \mathrm{~nm}$ and $\lambda=900 \mathrm{~nm}$ wavelengths. With respective BARC in place, the photodiode's quantum efficiency (QE) approaches $100 \%$ for $\lambda=750 \mathrm{~nm}$ with $20 \mu \mathrm{m}$ and $30 \mu \mathrm{m}$ iEPI thickness and for $\lambda=900 \mathrm{~nm}$ with $30 \mu \mathrm{m}$ iEPI reaching also a maximum spectral response of $0.63 \mathrm{~A} / \mathrm{W}$ at $800 \mathrm{~nm}$. QE of $72 \%$ could be achieved at $425 \mathrm{~nm}$. The leakage current varies from $3.5 \mathrm{pA}$ for $20 \mu \mathrm{m}$ iEPI thickness to $10 \mathrm{pA}$ for $30 \mu \mathrm{m}$ at $1 \mathrm{~V}$ reverse biasing for $365 \mu \mathrm{m}$ circular PIN photodiode.
\end{abstract}

Keywords: intrinsic epitaxial substrate; PIN photodiode; spectral responsivity; leakage current; capacitance

\section{Introduction}

A photodiode is a device based on a p-n junction collecting photocurrent generated in the substrate. The combination of reverse biasing and large depletion area are necessary for improving the quantum efficiency of the photodiode [1] because of the reduction of the transit time of the carriers in the substrate. A way to enlarge the depletion area of the photodiode is the use of low-doped intrinsic material thus forming a PIN photodiode. The low-doped intrinsic material additionally reduces the recombination rate of the photo carriers, thus improving the efficiency of the device. The photodiode can be tuned to be sensitive to specific wavelengths by using appropriate p-n junctions as well as a Bottom Anti-Reflective Coating (BARC) layer of wavelength dependent thickness. The impact of the different intrinsic p-type EPI layer thicknesses and photodiode designs are analyzed with respect to optical and electrical performances of circular and square photodiodes.

\section{Materials and Methods}

One type of PIN photodiodes [2] is constructed with a shallow highly doped $\mathrm{n}+$ type well in intrinsic p-type EPI (iEPI) (Figure 1a), hereafter called nPD. Another type optimized for blue wavelengths uses deep n-type well with a counter-doped shallow p-type well, thus making the 
photodiode less sensible to red and near infrared wavelengths (Figure 1b), hereafter called bluePD. By shortening deep n-type well and substrate, the photocurrent generated in the iEPI recombines and does not contribute to the photocurrent collected by the shallow p-well layer.
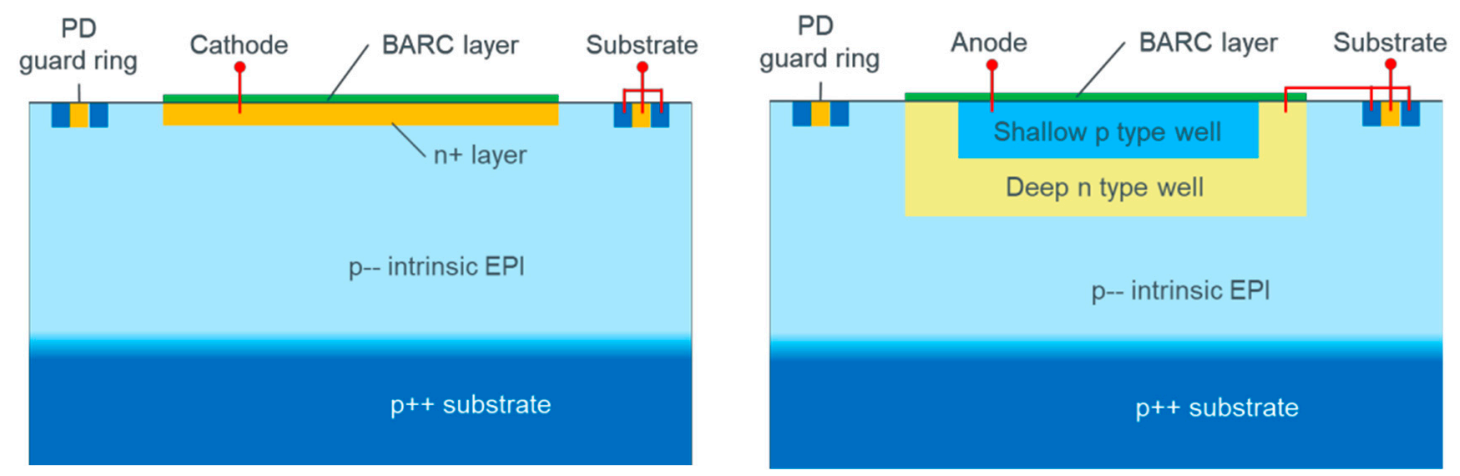

Figure 1. PIN photodiodes schematics where epitaxial intrinsic p-type layer is grown on low resistive substrate, substrate and guard contacts are placed at the silicon surface. (a) illustrates PIN photodiode with an n+ layer used as photocurrent collecting area (nPD). (b) optimized blue photodiode in which the photocurrent collecting area is a shallow p-type well inserted in a deep n-type well electrically connected to substrate (bluePD).

All photodiodes have been processed in an industry standard $350 \mathrm{~nm}$ high-voltage CMOS technology. The photodiodes' layout permits a direct pad access to anodes and cathodes without any signal amplification circuit. Photodiodes' optical characteristics are measured by guiding light from a tunable light source operating from $400 \mathrm{~nm}$ to $900 \mathrm{~nm}$ wavelengths through a multimode fiber placed above the photodiodes and measuring generated photocurrent on the different pads.

\section{Results}

Figure 2a presents the impact of the iEPI layer thicknesses and the BARC layers thicknesses on spectral responsivity (SR) for circular and square nPDs. Larger iEPI thickness increases the spectral response in near infrared wavelengths reaching a responsivity of $0.63 \mathrm{~A} / \mathrm{W}$ at $800 \mathrm{~nm}$ for a $30 \mu \mathrm{m}$ iEPI thickness while the BARC layer increases the local spectral response at its optimized wavelength. The spectral responsivity reaches $100 \%$ of the quantum efficiency for BARC layers optimized for $\lambda=750 \mathrm{~nm}$ and $\lambda=900 \mathrm{~nm}$ and $82 \%$ for layer optimized for $\lambda=425 \mathrm{~nm}$ wavelengths.

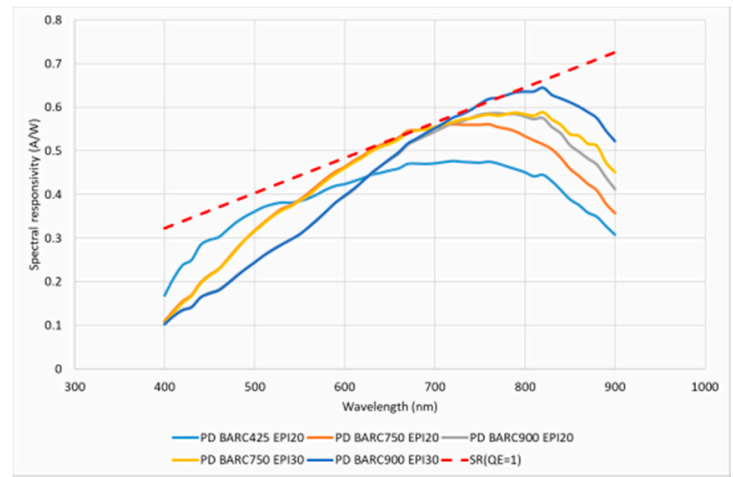

(a)

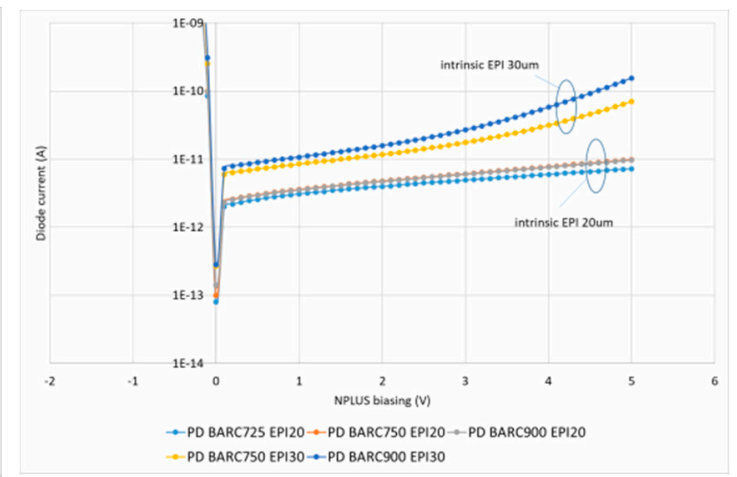

(b)

Figure 2. (a) iEPI thickness/BARC optimization of spectral responsivity as a function of wavelength for circular and square nPD. (b) Photodiode leakage current versus iEPI thickness and BARC optimization for circular nPDs Ø365 um.

The leakage current of circular nPD (Figures $2 b$ and $3 a, b$ ) is dependent on iEPI thickness, doping concentration, and the distance of photodiode to the guard ring [3]. The increase of the iEPI thickness increases the space charge region width around the pn junction thus increases the leakage current. 
The extension of the space charge region is more pronounced when the doping concentration of the EPI is closer to the intrinsic concentration $n_{i}$ which is confirmed by TCAD simulations shown in Figure 3a. At high bias voltage, we can see an increase of the leakage current due to the proximity of the photodiode guard ring consisting of a pnp substrate contact configuration where the $\mathrm{n}$ type contact layer is used for the reduction of photodiode to photodiode electrical and optical crosstalk. This configuration generates a lateral npn bipolar with the photodiode which increases the leakage current of the photodiode. In Figure 3b, we can observe that the leakage current is linearly dependent on the photodiode diameter or perimeter.

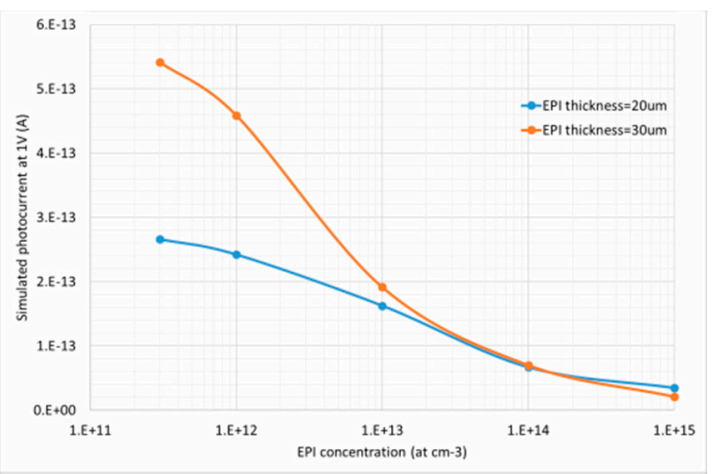

(a)

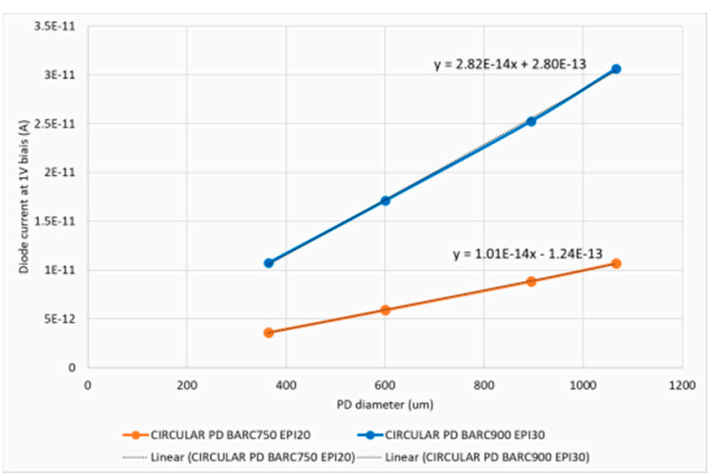

(b)

Figure 3. (a) TCAD simulation of nPD current magnitudes for various iEPI concentration on two EPI thicknesses. (b) Measured leakage current magnitude as a function of photodiode diameter.

The capacitance of the circular nPD is also dependent on the iEPI thickness and photodiode size (Figure 4a,b). The nPD capacitance is bounded by the iEPI thickness because of the low resistive substrate placed below the iEPI layer that is blocking the extension of the space charge width in depth of the photodiode. The circular photodiodes capacitance is linearly dependent to the surface of the photodiode while the impact of the BARC is limited (Figure $4 \mathrm{~b}$ ).

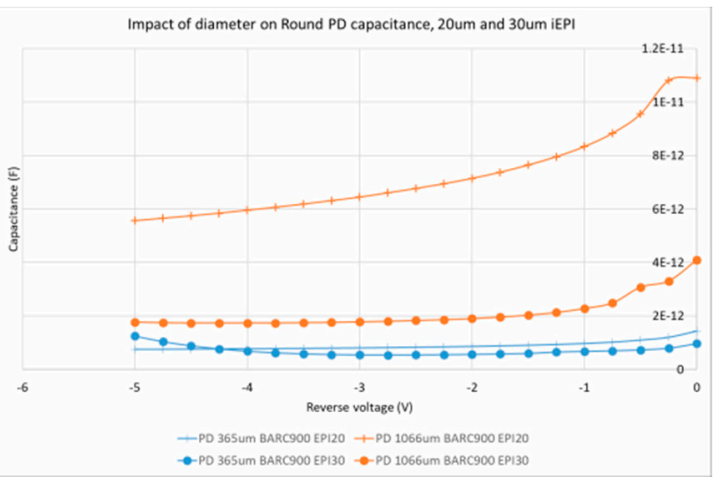

(a)

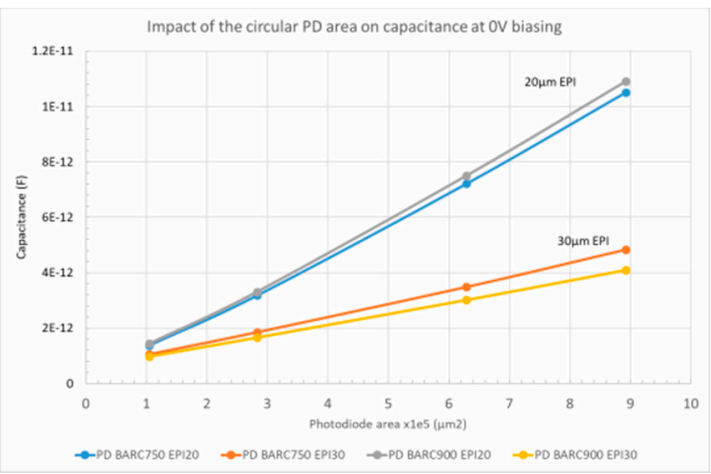

(b)

Figure 4. (a) Impact of scaling on circular nPD in $20 \mu \mathrm{m}$ and $30 \mu \mathrm{m}$ iEPI. (b). Evolution of capacitance at $0 \mathrm{~V}$ versus area of circular $\mathrm{nPD}$.

In addition to circular PIN photodiodes, square PIN photodiodes with a fixed single photodiode geometry of $200 \mu \mathrm{m} \times 200 \mu \mathrm{m}$ are placed. We also processed arrays of 16 square photodiodes $(4 \times 4$, each $200 \mu \mathrm{m} \times 200 \mu \mathrm{m}), 49$ square photodiodes $(7 \times 7)$ and 121 square photodiodes $(11 \times 11)$. The cathodes of all the photodiodes placed in an array are connected together. For arrays, the pnp guard ring is only placed around the array while each blocks have only a p type substrate contact.

Figure 5a shows the capacitance of a single square photodiode with $20 \mu \mathrm{m}$ and $30 \mu \mathrm{m}$ iEPI thicknesses. As observed for circular photodiodes, the capacitance is lowered when the iEPI thickness is increased. However, because of the smaller size of the squared photodiode, the space charge region of the photodiode reaches the $\mathrm{n}$ contact of the guard ring and thus limits the capacitance of the 
photodiode between $0 \mathrm{~V}$ and $-1.5 \mathrm{~V}$ for $30 \mu \mathrm{m}$ iEPI. This limitation is not observed for arrays. Figure $5 b$ shows the evolution of the capacitance of the square PIN photodiode versus the number of photodiodes placed in an array. The capacitance is varying linearly to the number of block. As each block has a fixed geometry, the capacitance is varying linearly to the surface of the array.

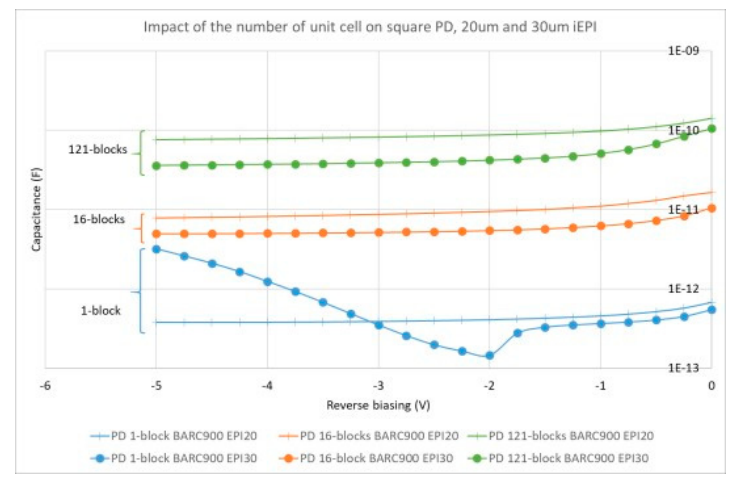

(a)

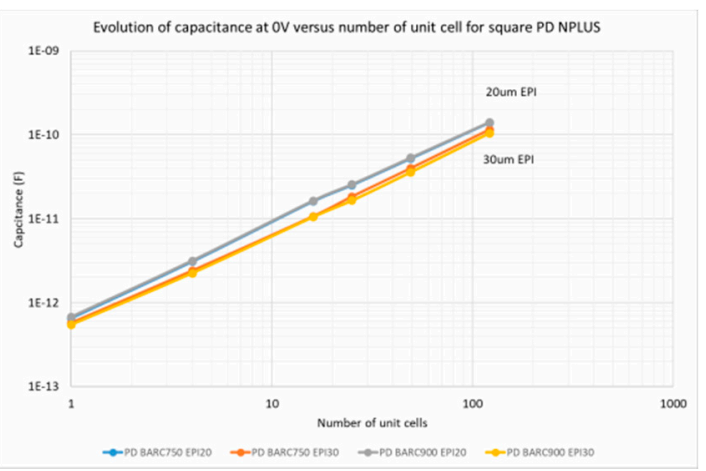

(b)

Figure 5. (a) Impact of scaling of square nPDs in $20 \mu \mathrm{m}$ and $30 \mu \mathrm{m}$ iEPI on the capacitance. (b) Evolution of capacitance at $0 \mathrm{~V}$ versus number of unit cells for square nPDs.

In the case of optimized bluePD (Figure 1b), the impact of iEPI thickness on the spectral responsivity curve is limited due to the internal collection of photocurrent while the application of a BARC layer optimized at $\lambda=425 \mathrm{~nm}$ increases the spectral current collected for short wavelengths (Figure 6a). Because of the internal structure of the bluePD, the leakage current is not dependent on the iEPI thickness and is around $10 \mathrm{pA}$ at a biasing below $-1 \mathrm{~V}$ for a $150 \mu \mathrm{m} \times 150 \mu \mathrm{m}$ photodiode size designed in a square configuration (Figure $6 b$ ).

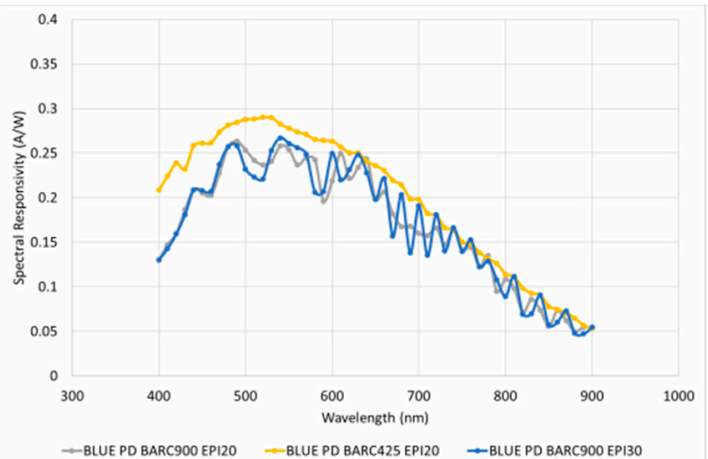

(a)

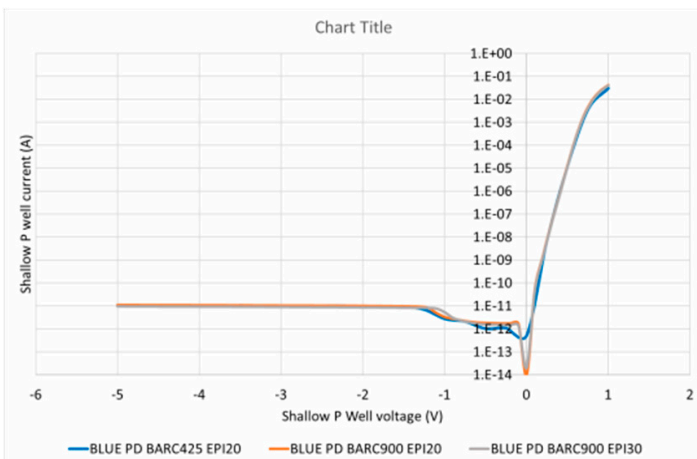

(b)

Figure 6. (a) Spectral responsivity for bluePD as a function of iEPI thickness and BARC optimization. (b) bluePD leakage current versus iEPI thickness and BARC optimization.

\section{Summary}

In this paper, we have presented the impact of low-doped intrinsic p-type epitaxial (iEPI) thicknesses $(20 \mu \mathrm{m}$ and $30 \mu \mathrm{m})$ and bottom antireflection coating (BARC) on the optical (spectral responsivity) and electrical (leakage current and capacitance) performances of various PIN photodiodes. PIN photodiodes ( $\mathrm{n}+$ in intrinsic $\mathrm{p}$ - epitaxial layer) reach a spectral responsivity of 0.63 $\mathrm{A} / \mathrm{W}$ and $100 \%$ quantum efficiency with the deposition of BARC layers optimized for $\lambda>750 \mathrm{~nm}$ in combination with $30 \mu \mathrm{m}$ iEPI thickness. The leakage current is larger with thicker iEPI thickness and varies from $3.5 \mathrm{pA}(20 \mu \mathrm{m}$ iEPI) to $10 \mathrm{pA}(30 \mu \mathrm{m}$ iEPI) for a circular PIN photodiode $(\varnothing 365 \mu \mathrm{m})$. Their capacitance is reduced for larger iEPI thickness and varies from $0.97 \mathrm{pF}(20 \mu \mathrm{m}$ iEPI) to $1.43 \mathrm{pF}$ (30 $\mu \mathrm{m}$ iEPI). Optimized blue photodiodes are not sensible to iEPI thicknesses and have a leakage current of $10 \mathrm{pA}$ for a $150 \mu \mathrm{m} \times 150 \mu \mathrm{m}$ square photodiode. 


\section{References}

1. Sze, S.M.; Ng, K.K. Physics of Semiconductor Devices, 3rd ed.; Wiley: Hoboken, NJ, USA, 2007; pp. 671-682.

2. Jonak-Auer, I.; Teva, J.; Park, J.M.; Jessenig, S.; Kailbauer, P.; Wachmann, E. New integration concept of PIN photodiodes in $0.35 \mu \mathrm{m}$ CMOS technologies. Proc. SPIE 2012, 8431, doi:10.1117/12.922268.

3. Teva, J.; Jessenig, S.; Jonak-Auer, I.; Schrank, F.; Wachmann, E. Dark current study for CMOS fully integrated-PIN-photodiodes. Proc. SPIE 2011, 8073, doi:10.1117/12.886985

(C) 2018 by the authors. Licensee MDPI, Basel, Switzerland. This article is an open access article distributed under the terms and conditions of the Creative Commons Attribution (CC BY) license (http://creativecommons.org/licenses/by/4.0/). 